\title{
Metabolic acidosis in a patient with metformin overdose
}

\author{
I Ebrahim, MB ChB; M Blockman, BPharm, MB ChB, PGDip Int Res Ethics, MMed \\ Division of Clinical Pharmacology, Department of Medicine, Faculty of Health Sciences, University of Cape Town, South Africa
}

Corresponding author: I Ebrahim (ebrism003@myuct.ac.za)

We report a rare fatal case of acute metformin overdose in a 19-year-old woman.

S Afr Med J 2017;107(2):110-111. DOI:10.7196/SAMJ.2017.v107i2.10971

A 19-year-old woman presented to a district-level emergency centre (EC) 1 hour after an intentional 'unquantifiable' metformin overdose. Clinical examination at that time was unremarkable except for a respiratory rate (RR) of 28 breaths per minute (bpm). No specific toxidrome was identified.

In the EC, 6 hours post metformin ingestion, she appeared restless and complained of severe abdominal pain for which ranitidine, hyoscine butylbromide and lorazepam were administered orally. The patient was observed overnight in the EC. Eighteen hours post ingestion her tachypnoea worsened to $40 \mathrm{bpm}$ and she developed hypoglycaemia, with a finger prick blood glucose reading of $1.8 \mathrm{mmol} / \mathrm{L}$. Venous blood gas findings taken at room temperature at this time are shown in Table 1.

Calculation of the anion gap was not possible because lactate and chloride values were not readily available. Based on the patient history and biochemical findings, a diagnosis of metabolic acidosis secondary to acute metformin overdose was made.

Infusions of $5 \%$ dextrose $\mathrm{w} / \mathrm{v}$ and $0.5 \%$ sodium bicarbonate were initiated separately, and the patient was immediately transferred to a secondary level hospital. Ongoing hypoglycaemia and worsening tachypnoea, accompanied by a drop in Glascow Coma Scale (GCS) to $12 / 15$, occurred en route and $50 \mathrm{ml}$ of a $50 \%$ dextrose bolus was administered.

The patient developed a prolonged QT interval which precipitated cardiac arrest, with a GCS of $4 \mathrm{~T} / 15$ requiring full resuscitation including inotropes. Arterial blood gas findings on 30\% oxygen at this time revealed a metabolic acidosis as shown in Table 2. At that time her creatinine was $235 \mathrm{mmol} / \mathrm{L}$ and the calculated increased anion gap was 27.3. The patient was then transferred to a tertiary hospital for dialysis and intensive care unit (ICU) care.

In the ICU, she had persistent refractory hypotension and worsening metabolic acidosis. Despite repeated doses of intravenous sodium bicarbonate and high-dose inotropic support, the GCS and metabolic acidosis remained unchanged. The patient died 42 hours post overdose despite aggressive ICU support.

\section{Discussion}

Metformin is a dimethylbiguanide. When prescribed in therapeutic doses for the management of type 2 diabetes mellitus, it is not known to cause hypoglycaemia. It is currently the only biguanide derivative available for this purpose. Two biguanides, phenformin and buformin, were removed from the market in the 1970s owing to their unpredictable association with lactic acidosis. ${ }^{[1,2]}$

Sixty percent of ingested metformin is absorbed from the gastrointestinal tract (GIT), not metabolised, and is dependent
Table 1. Venous blood gas on room air

\begin{tabular}{lllll}
\hline $\mathbf{p H}$ & $\mathbf{p C O}_{2}$ & $\mathbf{p O}_{2}$ & $\mathbf{H C O}_{3}$ & Base excess \\
\hline 6.9 & $11.7 \mathrm{kPa}$ & $8.9 \mathrm{kPa}$ & $23 \mathrm{mmol} / \mathrm{L}$ & $3 \mathrm{mmol} / \mathrm{L}$
\end{tabular}

Table 2. Arterial blood gas on $30 \%$ oxygen on arrival at the secondary hospital

\begin{tabular}{lllll}
\hline $\mathbf{p H}$ & $\mathbf{p C O}_{2}$ & $\mathbf{p O}_{2}$ & $\mathrm{HCO}_{3}$ & Base excess \\
\hline$<6.8$ & $1.5 \mathrm{kPa}$ & $22 \mathrm{kPa}$ & Not reported & Not reported
\end{tabular}

Table 3. Prescribed drugs associated with type $B$ hyperlactataemia $^{[5]}$

\begin{tabular}{ll}
\hline Drug class & Drug \\
\hline Lipid lowering & Statins \\
Analgesics & Fibrates \\
& Aspirin \\
Anticonvulsants & Paracetamol \\
Biguanides & Sodium valproate \\
Anti-arrhythmic & Metformin \\
Psychotropic & Amiodarone \\
& Fluoxetine \\
& Amitriptyline \\
& Chlorpromazine \\
Antiretroviral & Haloperidol \\
& Stavudine \\
& Didanosine \\
& Zidovudine
\end{tabular}

on glomerular filtration for elimination. It is excreted in urine, therefore reduced estimated glomerular filtration rate will affect its excretion. Its mechanism of action is complex, including reduction of intestinal glucose absorption, inhibition of hepatic gluconeogenesis and enhanced peripheral insulin sensitivity. ${ }^{[1,3]}$

Lactate is a byproduct of glucose and amino acid metabolism. Hyperlactataemia arises due to increased production or reduced metabolism of lactate. The condition is broadly classified into two types: ${ }^{[4]}$

- Type A: increased lactic acid in ischaemic tissue

- Type B: normal tissue perfusion in the presence of a mitochondrial 


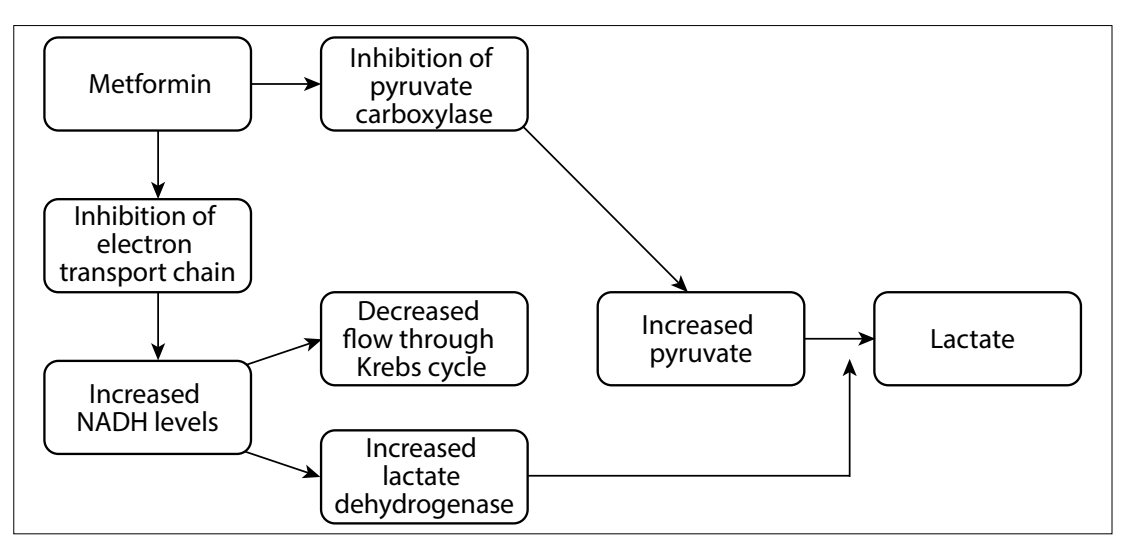

Fig. 1. Proposed mechanism for metformin-induced hyperlactataemia. $(\mathrm{NADH}=$ nicotinamide adenine dinucleotide, reduced form.)

defect (inherent or acquired secondary to pharmacotherapy). (Table 3)

Metformin-associated lactic acidosis (MALA) is a form of type B hyperlactataemia with a high anion gap. The estimated rate of MALA ranges between 3 and 9 cases per 100000 patient-years with a mortality $>50 \%{ }^{[6,7]}$

Metformin inhibits gluconeogenesis by direct inhibition of pyruvate carboxylase, which causes pyruvate to accumulate and an increase in lactate production. It also inhibits oxidative phosphorylation, impairing generation of $\mathrm{NAD}^{+}$from $\mathrm{NADH}$ by the mitochondria. This increase in $\mathrm{NADH}$ further favours conversion of pyruvate into lactate through inhibition of pyruvate dehydrogenase (Fig. 1). ${ }^{[8]}$

The resultant acidosis predisposes to dysrhythmias and diminished response to catecholamines. ${ }^{[9,10]}$ This may explain why
Metformin overdose should be treated immediately. The drug is extensively excreted renally and therefore haemodialysis is ideal in acute overdose for effective removal of both metformin and circulating lactate. ${ }^{[1,2,12]}$

\section{Conclusion}

Acute metformin overdose is rare and potentially life threatening. Severe metabolic acidosis hypoglycaemia and cardiovascular collapse are the main clinical features. Patients should be treated promptly in a critical care unit, with early consideration for haemodialysis and cautious use of intravenous sodium bicarbonate.

1. Spiller HA. Management of antidiabetic medications in overdose. Drug Saf 1998;19(5):411-424.

our patient had refractory hypotension despite high-dose inotropic support.

The management goals of MALA include restoration of acid-base status, removal of absorbed metformin and support of cardiovascular functions. ${ }^{[1]}$ Knowledge of the pharmacokinetic properties of metformin is essential in formulating the management plan. Metformin is partially absorbed through the gastrointestinal tract, with the unabsorbed portion binding to the intestinal wall. ${ }^{[3]}$ Activated charcoal is theoretically capable of binding the unabsorbed metformin ${ }^{[1]}$ and should be administered early if the patient is awake and has a protected airway.

Management of lactic acidosis with the use of sodium bicarbonate $\left(\mathrm{NaHCO}_{3}\right)$ is controversial despite its regular use. ${ }^{[1,2,9]}$ Theoretical disadvantages are a leftward shift of the oxygen dissociation curve, worsening intracellular acidosis, rebound metabolic alkalosis and fluid overload. ${ }^{[1,2,9]}$
2. Heaney D, Majid A, Junor B. Bicarbonate haemodialysis as a treatment of metformin overdose. Nephrol Dial Transplant 1reatment of metform

3. Scheen AJ. Clinical pharmacokinetics of metformin. Clin Scheen AJ. Clinical pharmacoki
Pharmacokinet 1996;30(5):359-371.

Cohen RD, Woods H. Clinical and Biochemical Aspects of Cohen RD, Woods H. Clinical and Biochemical Aspects
Lactic Acidosis. Oxford: Blackwell Scientific Publication, 1976. 5. Neustadt J, Pieczenik SR. Review of medication-induced mitochondrial damage and disease. Mol Nutr Food Res 2008;52(7):780-788. https://dx.doi.org/10.1002/mnfr.200700075

6. Stang M, Wysowski DK, Butler-Jones D. Incidence of lactic acidosis in metformin users. Diabetes Care 1999;22(6):925-927.

7. Lalau JD, Race JM. Lactic acidosis in metformin-treated metformin concentrations. Drug Saf 1999;20(4):377-384.

8. Dembo AJ, Marliss EB, Halperin ML. Insulin therapy in phenformin-associated lactic acidosis. Diabetes 1975;24(9):2835. https://dx.doi.org/10.2337/diab.24.9.791

9. Velissaris D, Karamouzos V, Ktenopoulos N, et al. The use of sodium bicarbonate in the treatment of acidosis in sepsis: A
sodissaris D, Karamouzos sodium bicarbonate in the treatment of acidosis in sepsis: A
literature update on a long term debate. Crit Care Res Pract literature update on a long term debate. Crit Care Res Pact
2015;2015:605830. http://dx.doi.org/10.1155/2015/605830

10. Orchard $\mathrm{CH}$, Cingolani HE. Acidosis and arrhythmias in cardiac muscle. Cardiovasc Res 1994;28(9):1312-1319.

11. Spiller HA, Sawyer TS. Toxicology of oral antidiabetic medications. Am J Health Syst Pharm 2006;63(10):929-938. https://dx.doi.org/10.2146/ajhp050500

12. Nguyen HL, Concepcion L. Metformin intoxication requiring dialysis. Hemodial Int 2011;15(1):S68-71. https://dx.doi. org/10.1111/j.1542-4758.2011.00605.x

Accepted 19 October 2016. patients. Prognostic value of arterial lactate levels and plasma 